\title{
Is Environment Really A Function?
}

\author{
Gregor Burkhart
}

Published online: 12 December 2013

(C) European Union 2013

I congratulate David Foxcroft for this fine paper "Can prevention classification be improved by considering the function of prevention?" In essence, it proposes, in addition to the classification into universal, selective, and indicated ("forms"), another axis, where environmental, developmental, and informational approaches are introduced as "functions." In this system, environmental prevention would no longer be a fourth category complementing universal, selective, and indicated prevention, but another functional dimension, pervading transversally the three forms of prevention. When David presented his model at the Second Annual Meeting of the European Society for Prevention Research, I was puzzled, and since then, have continued pondering whether this proposal is really preferable to the model we have been promoting within the EU.

A number of David's critical comments on the existing model present me with problems as follows:

1. David makes a point that in the current model, environmental prevention would overlap with universal prevention, and even with selective and indicated prevention, because it can have differential effects or may address certain groups only, such as minors. Taken to its logical conclusion - the argument that measures lack universality - then only mass media campaigns are universal, because all other approaches commonly labeled "universal" target sub-populations, like young people, working people, and so on. I had always understood from the literature that the defining characteristic of "universal" is that the vulnerability of the target population is not assessed

G. Burkhart $(\bowtie)$

European Monitoring Centre for Drugs and Drug Addiction (EMCDDA), Praca Europa 1, Cais do Sodré, 1249-289 Lisbon, Portugal

e-mail: Gregor.Burkhart@emcdda.europa.eu

URL: www.emcdda.europa.eu differentially. Besides, the model we have been using until now was not meant to consist of clear-cut side-by-side categories, but rather of spheres-within-spheres, similar to Bronfenbrenner's 1979 model of macro-, meso-, and microspheres of influence. Environmental prevention, here, is an outer sphere, which targets physical, social, economic, and virtual environments. In contrast to the other three forms, it does not target people directly (i.e., in direct interaction). Nevertheless, all four approaches obviously aim at behavioral change of individuals. Therefore, I do not see it as conceptually confusing that the outer sphere, in the form of family norms and socialization - as examples of environmental prevention-has effects on an individual child. Most of us would agree that all prevention strategies aim at ultimately changing individual behavior; the environmental ones by changing context, the other three by persuasion according to differing vulnerabilities and needs. That environmental strategies can be carried out within community strategies does also not constitute a violation of the category system: all four forms (or "spheres") of prevention can be carried out in all classical settings for prevention. Lastly, even if gun-control laws or age limits might affect only or mostly those most at risk, they are still applied to society and the population as a whole, unlike indicated approaches, which target selected individuals only.

2. David seems to interpret environmental prevention as restricted to regulation alone. However, non-coercive environmental influences on behavior can also be classed as environmental prevention, even if sometimes the borders with health promotion may be ill-defined. Examples are positive school or learning climate and the spatial and logistical arrangement of nightlife events. None of these imply changing behavior by persuasion.

3. I would not subscribe to the view that universal approaches "are generally more impactful on better off, lower risk population groups." Rather, it seems - when 
applying innovative statistical methodologies (Brown et al. 2011; Muthén et al. 2002) - that universal prevention can be more beneficial for the more vulnerable subgroups. Recent evaluations of universal prevention programs have shown such effects on the more vulnerable children in elementary school (Kellam et al. 2008); some selective family based programs seem to be more effective the more vulnerable the targeted families are (Kumpfer et al. 2008); a school-based program, evaluated in seven European countries under the EU-DAP trial, showed a more favorable effect on problematic drinking among students in underprivileged social environments (Caria et al. 2011); and the evaluation of an alcohol prevention program in both school and family settings combined (Koning et al. 2012) found that the more vulnerable subgroups within the universal target population benefited more from the intervention. This might be due to vulnerable subgroups adjusting their behavior to that of the "conventional" majority.

There is no disputing that mass media campaigns should not count as environmental prevention, specifically because they use persuasion and do not change context. Provided we agree that they are preventive at all, they might rather be seen as universal approaches.

These points hopefully illustrate that the existing model of environmental, universal, selective, and indicated prevention in concentric spheres (or "tiers") seems quite consistent, and that the alleged overlaps that would violate the model's rules can be explained.

However, and regardless of the argument defending the existing classification, I am deeply grateful to David Foxcroft for contributing to a more refined and pragmatic classification of prevention. It is particularly welcomed that David introduces three functional categories (environmental, developmental, and informational), which seem practical and helpful to further and more accurately differentiate prevention approaches within the existing categories. There is certainly a need for such functional categories.

I would like to express, though, a number of concerns and doubts, which David might want to address in order to increase the acceptance of the model proposed.

1. I was surprised that David's paper does not make reference to the recently published and helpful "COM-B system" (Michie et al. 2011), which proposes Capability, Opportunity and Motivation, as main constituents of Behavioral change. The three components are in detail as follows:

- Capability is distinguished between physical and psychological capability, the latter being the capacity to engage in the necessary thought processes through comprehension, reasoning, and so on.

- Opportunity is distinguished between physical opportunity afforded by the environment and social opportunity afforded by the cultural milieu that dictates the way that we think about things (e.g., the words and concepts that make up our language).

- Motivation is distinguished between reflective processes (involving evaluations and plans) and automatic processes (involving emotions and impulses that arise from associative learning, innate dispositions, or both).

In common with David's model, the COM-B system proposes "functions" (education, persuasion, incentivisation, coercion, training, restriction, environmental restructuring, modeling, enablement). However, I find it a relief that David manages to collapse almost this whole spectrum into only three functions, in particular, the clear distinction drawn between informational approaches and those beautifully defined as developmental: "intended to shape the socialization and development of young people as they grow and mature so that they are less susceptible to any such opportunities for risky behavior that are present in the environment."

2. My concern is that not only does David use "environmental" as a function instead of a form, but above all, that he limits it to "interventions that aim to limit the availability of maladaptive behavior opportunities, through systemwide policies, restrictions, and actions." This seems - as said above - too limited. If we conceive environmental prevention as all approaches that act on context but not through persuasion, such a category should not only embrace the COM-B functions "coercion" and "restriction," but also "environmental restructuring" and maybe "enablement" - that is not only regulatory aspects.

3. In addition, it seems to me that the proposed three functional categories have some fault lines across their boundaries. According to David's model, developmental prevention would operate through the "social learning of acceptable and unacceptable behaviors." Is it not quite conservative to assume that the adoption of acceptable behavior is achieved only through social learning by focusing on the development of skills? Have we not seen that notions of acceptable and unacceptable behaviors are conveyed also (or more) through descriptive and injunctive norms (often through environmental strategies)? If we do not discard litter on clean streets (descriptive norms) or because we saw somebody else putting our dropped litter in a bin (injunctive norms), it is certainly not because of acquired skills. Because of these normative mechanisms, as in the broken window theory (Kelling and Wilson 
1982), social learning by observation — or broader: socialization - would also need to be classified as environmental prevention. So, there is confusion as well. Furthermore, the effectiveness of family norms and monitoring is mostly not due to the ultimate target group (young people) having acquired any skills. It is the parents' skills in creating protective, normative, and nurturing family environments that lead to positive outcomes. According to van der Vorst et al. (2006, 2007), talking to offspring about alcohol can be counterproductive, while parental monitoring is protective. I would only consider the former as developmental, but the latter as environmental, as it does not imply persuasion or skills training of the offspring.

4. Important processes that shape our behavior are neither related to cognition skills nor to coercion. It seems to me that the model proposed by David does not acknowledge this sort of behavioral determinant, which is - by the way - also utterly ignored by prevention professionals. To which category would aspects of "motivation" from the COM-B model such as emotion, impulse, and association belong? In the existing "spherical" model, we could fit unconscious cues and affordances very well under environmental prevention. David himself is tutoring research about affordances (spatial and other structural arrangements in bars, sizes of beer glasses), and R. Wiers for example on implicit cognitions (Wiers et al. 2004), but it is difficult to find them in this model where "environmental" is only "system-wide policies, restrictions, and actions; for example, legal restrictions, economic (dis)incentives or situational crime prevention."

I always appreciated that the current classification of prevention allowed space for interventions that target the unconscious, associative, and imitative processes of socialization: under the sphere of "environmental prevention," i.e., where non-persuasive strategies have been subsumed.

David's proposal of introducing the concept of functions into our classification of prevention adds many useful and practical innovations that, by taking into account its contents and not only its form, can make prevention more meaningful. Nevertheless, I would still contend that we can introduce three (or more) functions into our current model, without having to define environmental prevention as a function instead of a form. Furthermore, non-conscious behavioral determinants need to be recognized in such a model. Maybe another look at the functions proposed by Michie et al. (2011) could lead us further. For example, training, incentivisation, and modeling might serve to better define "developmental." Likewise, restructuring, coercion, restrictions, and maybe enablement could be summarized in a function that is not related to either persuasion, acquiring skills, or information. Maybe "normative restructuring" or simply "nudging" is a term that would subsume these functions in one category that represents also the role of affordances and other non-conscious processes, but without confounding with the form of "environmental prevention." It seems to me that such a function would quite plausibly pervade the four spheres of prevention, as for example coercion in regulatory legislation, restructuring in how vulnerable neighborhoods can be re-urbanized; and the Good Behavior Game (Kellam et al. 2008) is an example of how nudging works in universal prevention. Changing associations learned, attention biases, and implicit attitudes would be an example applied in indicated prevention. In other words, we ought to name a third function to comprise all those aspects of prevention that target the unconscious, associative and context. Many of these happen to occur in the environmental "sphere." Other discussants might offer a better term than the ones I have proposed ("normative restructuring" or "nudging").

And yet, I still see environmental prevention rather as a form than a function of prevention, because when we respond to the question "what is your target?" we could point to any of the following: the context (environmental prevention), people in general (universal prevention), vulnerable groups (selective prevention), or vulnerable people (indicated prevention). If a function answers to the question "how to act?" or "what for?" then I am not convinced that a non-specialist would easily identify "environment" as a function, comparable to information, skills training, coercion, persuasion or... nudging.

However, we need to integrate David's very valuable point that shaping context is also a function; and that, in any case, functions need to be considered and defined in our model of prevention, if it is to become more useful.

This ongoing discussion is very important and I am grateful to be able to participate in it.

\section{References}

Bronfenbrenner, U. (1979). The ecology of human development: Experiments by nature and design. Cambridge, MA: Harvard University Press.

Brown, C. H., Sloboda, Z., Faggiano, F., Teasdale, B., Keller, F., Burkhart, G., et al. (2011). Methods for synthesizing findings on moderation effects across multiple randomized trials. Prevention Science, 14, 144-156.

Caria, M. P., Faggiano, F., Bellocco, R., \& Galanti, M. R. (2011). The influence of socioeconomic environment on the effectiveness of alcohol prevention among European students: A cluster randomized controlled trial. BMC Public Health, 11, 312.

Kellam, S. G., Brown, C. H., Poduska, J. M., Ialongo, N. S., Wang, W., Toyinbo, P., et al. (2008). Effects of a universal classroom behavior management program in first and second grades on young adult behavioral, psychiatric, and social outcomes. Drug and Alcohol Dependence, 95, S5-S28.

Kelling, G. L., \& Wilson, J. Q. (1982, March 1). Broken windows-The police and neighborhood safety. Boston: The Atlantic. 
Koning, I. M., Verdurmen, J. E. E., Engels, R. C. M. E., van den Eijnden, R. J. J. M., \& Vollebergh, W. A. M. (2012). Differential impact of a Dutch alcohol prevention program targeting adolescents and parents separately and simultaneously: Low self-control and lenient parenting at baseline predict effectiveness. Prevention Science, 13, 278-287.

Kumpfer, K. L., Pinyuchon, M., De Melo, A. T., \& Whiteside, H. (2008). Cultural adaptation process for international dissemination of the strengthening families program. Evaluation and the Health Professions, 31, 226-239.

Michie, S., van Stralen, M. M., \& West, R. (2011). The behaviour change wheel: A new method for characterising and designing behaviour change interventions. Implementation Science, 6, 42.

Muthén, B., Brown, C. H., Masyn, K., Jo, B., Khoo, S. T., Yang, C. C., et al. (2002). General growth mixture modeling for randomized preventive interventions. Biostatistics, 3, 459-475.
Van der Vorst, H., Engels, R. C. M. E., Dekovic, M., Meeus, W., \& Vermulst, A. (2007). Alcohol-specific rules, personality and adolescents' alcohol use: A longitudinal person-environment study. Addiction, 102, 1064-1075.

Van der Vorst, H., Engels, R. C. M. E., Meeus, W., \& Deković, M. (2006). The impact of alcohol-specific rules, parental norms about early drinking and parental alcohol use on adolescents' drinking behavior. The Journal of Child Psychology and Psychiatry and Allied Disciplines, 47, 1299-1306.

Wiers, R. W., de Jong, P. J., Havermans, R., \& Jelicic, M. (2004). How to change implicit drug use-related cognitions in prevention: A transdisciplinary integration of findings from experimental psychopathology, social cognition, memory, and experimental learning psychology. Substance Use \& Misuse, 39, $1625-1684$ 